\title{
SCIENTIFIC ACHIEVEMENTS OF PROFESSOR JACEK RYCHLEWSKI \\ (1947-2003)
}

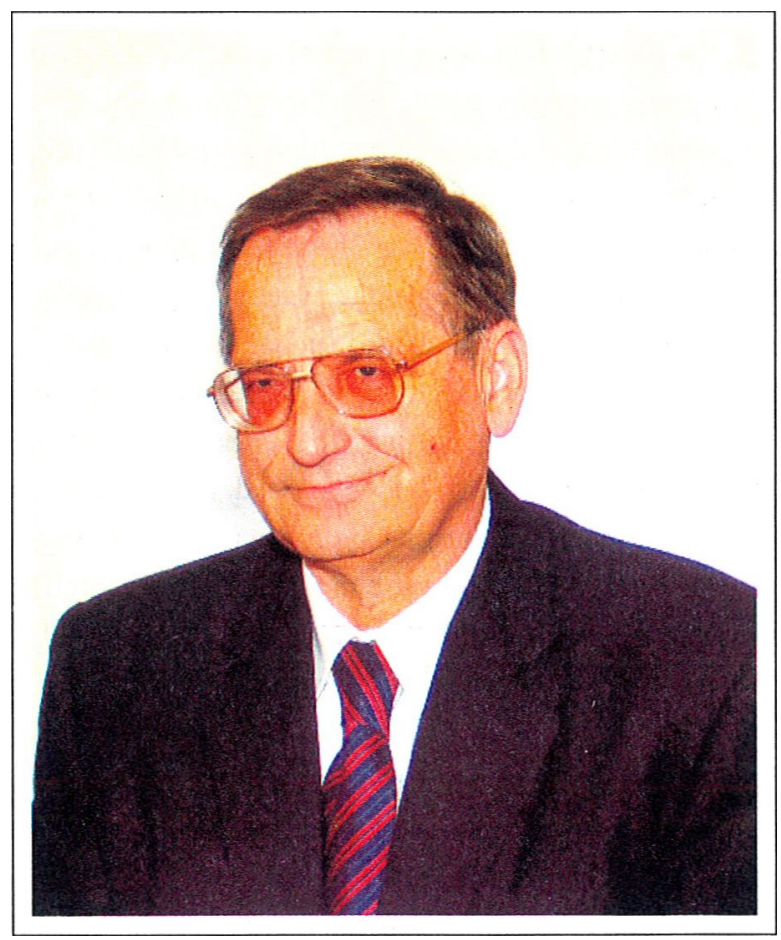

Jacek Rychlewski was born in 1947 at Poznań, Poland. He received a Master's Degree in Mathematics in 1969 a second Master's Degree, in Chemistry, in 1970, both degrees from the Adam Mickiewicz University in Poznań. In 1970 Dr. Rychlewski became a research assistant at the Department of Chemistry of this University. In 1971 he moved to the University of Warsaw and joined Włodzimierz Kołos group in the Department of Chemistry there. In 1975 he received the $\mathrm{PhD}$ in chemistry for his dissertation, "Interaction between the $B^{l} \Sigma_{u}^{+}$and $C^{l} \Pi_{u}$ states of the hydrogen molecule". In 1974 he returned to the A. Mickiewicz University in Poznań where he became an associate professor in 1986, and a full professor of theoretical chemistry in 1992. Since 1995, he was also made a full professor of computational chemistry at the Institute for Bioorganic Chemistry of the Polish Academy of Sciences in Poznań.

Since 1974 Jacek Rychlewski worked at various foreign scientific institutions including the University of Florida in Gainesville (USA), University of Sheffield (United Kingdom), University of North Carolina at Chapel Hill (USA), and the University of Wyoming at Laramie (USA). Professor Rychlewski's list of scientific publications contains over 100 original papers devoted to a broad range of problems in theoretical and computational chemis- 
try and in molecular physics. He made important contributions to such diverse fields as: the theory of molecular structure, the theoretical spectroscopy of the hydrogen molecule, the theory of excited states and interactions between a molecule and external electromagnetic fields, the theory of adiabatic approximation, applications of explicitly correlated functions in chemistry and physics, the theory of an atom in a molecule, the theory of molecular effects in $\beta$-decay, the theory of the hydrogen bond, the dimerization of $\alpha$-hydroxyamide groups, conformational studies of tartaric acid derivatives, high-performance computing, and the parallelization of computational studies on both homogeneous and heterogeneous systems.

His most eminent scientific achievement was the high-accuracy quantum mechanical treatment of two-, three-, and four-electron molecular systems. By developing new approximate schemes at the Born-Oppenheimer, adiabatic, nonadiabatic, and relativistic levels of description he achieved accuracy matching the best contemporary experiments. These works provided a wealth of theoretical data to inspire further developments of experimental techniques. Together with his co-workers he developed new methods based on explicitly correlated functions: the generalized James-Coolidge and explicitly correlated Gaussian functions. Using these methods he obtained the most accurate theoretical results ever found in molecular structure theory for such systems as $\mathrm{H}_{2}$ in the ground and excited states, $\mathrm{HeH}^{+}$, $\mathrm{H}_{3}{ }^{+}, \mathrm{H}_{3}, \mathrm{H}_{2}{ }^{+}, \mathrm{LiH}$, and $\mathrm{He}_{2}$. These results serve as the reference data both for theory and experiment.

Another area of Rychlewski's activity was the theory of interaction of molecules with external fields. He made an important contribution to this theory by performing very accurate calculations of molecular parameters such as the polarizability, the magnetizability, the rotational g-factor, as well as the nuclear shielding for the hydrogen molecule in its ground and excited states. His most spectacular finding was the temperature-independent paramagnetism of the hydrogen molecule in its $B$ and $B^{\prime}{ }^{1} \Sigma_{u}^{+}$states. Besides the ground state of a hypothetical BH molecule the above states are the only well-documented molecular states with such a property. It was then suggested that such paramagnetism might be very common among the excited states of diatomic molecules. These properties, as calculated by Rychlewski, can also serve as reference data.

Recently, Rychlewski has been engaged in the theoretical study of the adiabatic approximation. In a series of papers he has shown that the double-minimum potential energy curves can appear as an exclusive result of adiabatic effects. This was found in two pairs of states of hydrogen molecule such as $h$ and $g$, and 4 s and $4 d^{3} \Sigma_{g}^{+}$. The same effect has been found very recently for the $r$ and $w 3 \Pi_{g}$ states of $\mathrm{H}_{2}$.

For his outstanding contributions Rychlewski was awarded both the the Scientific Award of City of Poznań and the Polish Chemical Society Prize. Recently, he also served as the advisory editor of Progress in Theoretical Chemistry and Physics published by the Kluwer Academic Publishers. He was one of the founders and editors of Computational Methods in Science and Technology and, as its executive editor, spent considerable effort in making this journal a valuable one, not only for local scientists, but also for the international scientific community. Rychlewski was also active at the international level. He was a member of Man- 
agement Committee of COST D9 and D26 Actions and coordinated the working group COSTD9/0013 devoted to theory of explicitly correlated wave functions and their applications to the theoretical chemistry and physics.

Professor Rychlewski devoted much of his talent and energy to educational and administrative activities in science. Since 1992 he was engaged in creating tools for computational chemistry and physics in supercomputing and networking centers in Poland. From 1993 until his death last year, he was the Chairman of the Council of Users of the Poznań Supercomputing and Networking Center. He was also the chairman of the PIONEER project for developing the computer science infrastructure for the Polish scientific community. He also served as the advisor for the scientific and innovative policy to the Minister of Science, Chairman of the State Committee for Scientific Research.

On May 27, 2003, at the age of only fifty five, Jacek Rychlewski passed away suddenly while working in his scientific laboratory on the book presenting the theory and application of explicitly correlated wave functions - the topic to which he devoted most of his life.

His death is a great loss for both the Polish and the international scientific communities.

\section{J. Komasa}

J. Weglarz

K. W. Wojciechowski 